\title{
Correlated two-electron transport: A principle for a charge pump
}

\author{
Qing-feng Sun, ${ }^{1,3}$ Hong Guo, ${ }^{1,3}$ and Jian Wang ${ }^{2}$ \\ ${ }^{1}$ Center for the Physics of Materials and Department of Physics, McGill University, Montreal, PQ, Canada H3A 2T8 \\ ${ }^{2}$ Department of Physics, The University of Hong Kong, Pokfulam Road, Hong Kong, China \\ ${ }^{3}$ International Center for Quantum Structures, Institute of Physics, Chinese Academy of Sciences, Beijing 100080, China
}

(Received 31 March 2003; published 16 July 2003)

\begin{abstract}
By considering a correlated two-electron transport (TET) process and using a diagrammatic analysis within the Keldysh nonequilibrium Green's-function formalism, we discuss a charge pump by which carriers are pumped from a contact with low chemical potential to another contact with a higher potential. The TET process involves two correlated incident electrons scattering and exchanging energy with each other. The process can redistribute the current over energies and it involves high empty states and/or low filled states of the Fermi liquid of the leads.
\end{abstract}

DOI: 10.1103/PhysRevB.68.035318

PACS number(s): 73.23.Hk, 73.40.Gk

In the past two decades, quantum electron transport through mesoscopic and nanoscopoic structures has received considerable attention. ${ }^{1}$ Charge transport theories for nanostructures typically consider the situation of an incident electron from a lead that is scattered in the device scattering region and transmitted to other leads or reflected back from where it came. The scattering region can be rather complicated and may involve electron-electron $(e-e)$, electronphonon, electron-impurity, and other interactions and scattering mechanisms. The scattering region itself can be a semiconductor nanostructure such as a quantum dot (QD), a carbon nanotube, or a single molecule. The scattering processes may involve exchange of energy or spin. While situations and physics can vary widely, charge transport in nanostructures has, so far, largely been considered as involving a single electron incident from a noninteracting lead of the device; it traverses through a complicated scattering region in which interactions occur and finally exits the device through the noninteracting leads. We will refer to them as single-electron tunneling or single-electron transport (SET) processes.

In this paper, we go one step further by investigating correlated two-electron tunneling and transport (TET) processes which, to the best of our knowledge, have not been studied before. In these processes, two incident electrons with energies $\epsilon_{1}$ and $\epsilon_{2}$ tunnel from the leads into the scattering region of a device, scatter with each other, and exchange energy (they could also scatter and exchange energy with other particles in the scattering region). Following the scattering event, they transmit to the outside world through the contacts, but with energies $\epsilon_{1}^{\prime}$ and $\epsilon_{2}^{\prime}$ which are different from the initial values $\epsilon_{1}, \epsilon_{2}$ due to energy exchange. If $\epsilon_{1}+\epsilon_{2}$ $=\epsilon_{1}^{\prime}+\epsilon_{2}^{\prime}$, i.e., the two incident electrons do not exchange energy with other particles, the transport process as a whole can be considered as elastic. In the TET process, the variation of electron number in the scattering region is always two: a situation that is different from SET. We show that such TET processes can indeed occur, and it affects measurable physical quantities such as charge current. In particular, TET induces a transport vertical flow-meaning carriers incident with energy $\epsilon_{1}$ exit at a different energy $\epsilon_{1}^{\prime}$, resulting to a nonconserved current density, i.e., $\Sigma_{n} j_{n}(\epsilon) \neq 0$. Using the characteristics of the correlated two-electron transport process, we design an interesting charge pump so that incident carriers can be pumped from a contact with low chemical potential to another contact having a higher potential.

We consider a QD coupled to two or three leads, described by the following Hamiltonian:

$$
\begin{aligned}
H= & \sum_{\alpha} \epsilon_{\alpha} d_{\alpha}^{\dagger} d_{\alpha}+U d_{\uparrow}^{\dagger} d_{\uparrow} d_{\downarrow}^{\dagger} d_{\downarrow}+\sum_{n, k, \alpha} \epsilon_{n k} a_{n k \alpha}^{\dagger} a_{n k \alpha} \\
& +\sum_{n, k, \alpha}\left[t_{n k} a_{n k \alpha}^{\dagger} d_{\alpha}+\text { H.c. }\right],
\end{aligned}
$$

where $a_{n k \alpha}^{\dagger}\left(a_{n k \alpha}\right)$ and $d_{\alpha}^{\dagger}\left(d_{\alpha}\right)$ are creation (annihilation) operators in lead $n$ and in QD, respectively. The QD includes two states and has an intradot Coulomb interaction $U$. The subscript $\alpha$ is the spin index, it may also indicate other quantum numbers. To account for a magnetic field, we let $\epsilon_{\uparrow}$ $\neq \epsilon_{\downarrow}$.

In the following we focus on investigating elastic TET by analyzing the behavior of current density $j_{n}(\epsilon)$ from lead $n$ to the QD. $j_{n}(\epsilon)$ relates to the current $I_{n}$ through $I_{n}$ $=\int j_{n}(\epsilon) d \epsilon$. We also define the electron occupation number density operator

$$
\hat{N}_{n}(\epsilon, \tau)=\sum_{k, \alpha} \int e^{i \epsilon t} a_{n k \alpha}^{\dagger}(\tau) a_{n k \alpha}(\tau+t)(d t / 2 \pi)
$$

$\hat{N}_{n}(\epsilon, \tau) d \epsilon$ describes the electron occupation number in lead $n$ in the energy range $\epsilon$ to $\epsilon+d \epsilon$ at time $\tau$. Current density $j_{n}(\epsilon)$ can be calculated from the time evolution of $\hat{N}_{n}(\epsilon, \tau): j_{n}(\epsilon)=-e\left\langle(d / d \tau) \hat{N}_{n}(\epsilon, \tau)\right\rangle$. This leads to (in units of $\hbar=1)^{2}$

$$
j_{n}(\epsilon)=-e \operatorname{Im} \sum_{\alpha} \frac{\Gamma_{n}(\epsilon)}{2 \pi}\left[2 f_{n}(\epsilon) G_{\alpha}^{r}(\epsilon)+G_{\alpha}^{<}(\epsilon)\right]
$$

where linewidth function $\Gamma_{n}(\epsilon) \equiv 2 \pi \Sigma_{k}\left|t_{n k}\right|^{2} \delta(\epsilon$ $\left.-\epsilon_{n k}\right) ; f_{n}(\epsilon)$ is the Fermi distribution function for lead $n ; G_{\alpha}^{r,<}(\epsilon)$ are the retarded and Keldysh Green's functions 
of the QD. ${ }^{3}$ With the use of the standard equation of motion technique, $G_{\alpha}^{r}(\epsilon)$ has already been solved in previous work: ${ }^{3,4}$

$$
G_{\alpha}^{r}(\epsilon)=\frac{1+U A_{\alpha} n_{\bar{\alpha}}}{\epsilon-\epsilon_{\alpha}-\Sigma_{\alpha}^{(0)}(\epsilon)+U A_{\alpha}\left(\Sigma_{-\alpha}^{a}+\Sigma_{-\alpha}^{b}\right)},
$$

where

$$
A_{\alpha}(\epsilon)=\left[\epsilon-\epsilon_{\alpha}-U-\Sigma_{\alpha}^{(0)}(\epsilon)-\Sigma_{-\alpha}^{(1)}(\epsilon)-\Sigma_{\alpha}^{(2)}(\epsilon)\right]^{-1} ;
$$

$\Sigma_{\alpha}^{(0)}$ is the lowest-order self-energy, $\Sigma_{\alpha}^{(1),(2), a, b}$ are the higher-order self-energies; ${ }^{3}$ and $n_{\alpha}=\operatorname{Im} \int(d \epsilon / 2 \pi) G_{\alpha}^{<}(\epsilon)$ is the intradot electron occupation number of state $\alpha$. It is worth mentioning that if temperature $T$ is lower than the Kondo temperature $T_{K}$, the solution of Eq. (2) has a Kondo resonance at the Fermi level which has been the subject of many previous studies. ${ }^{4}$

We investigate TET processes at a temperature higher than $T_{K}$. To this end we need to solve the the Keldysh Green's function $G_{\alpha}^{<}(\epsilon)$. Note that if one applies the commonly used ansatz for interacting lesser and greater self-energies, ${ }^{5}$ or using the large- $U$ limit noncrossing approximation $^{4,6}$ to solve $G_{\alpha}^{<}(\epsilon)$, the two-electron scattering will be lost in these approximations. Therefore a more precise analysis is needed in our problem and we proceed as follows. Introducing the intradot electron occupation number density operator $\hat{N}_{\alpha}(\epsilon, \tau)=\int e^{i \epsilon t} d_{\alpha}^{\dagger}(\tau) d_{\alpha}(\tau+t)(d t / 2 \pi)$ and due to the steady-state condition $\left\langle(d / d \tau) \hat{N}_{\alpha}(\epsilon, \tau)\right\rangle=0$, we have

$$
-e \sum_{n} \frac{i \Gamma_{n}}{2 \pi}\left\{G_{\alpha}^{<}(\epsilon)+f_{n}\left[G_{\alpha}^{r}(\epsilon)-G_{\alpha}^{a}(\epsilon)\right]\right\}=i_{\alpha}(\epsilon),
$$

where $i_{\alpha}(\epsilon)$ gives the intradot vertical flow $i_{\alpha}(\epsilon)$ $\equiv i e U \int e^{i \epsilon t}\left\{\left\langle d_{\alpha}^{\dagger}(0) d_{\bar{\alpha}}^{\dagger}(0) d_{\bar{\alpha}}(0) d_{\alpha}(t)\right\rangle-\left\langle d_{\alpha}^{\dagger}(0) d_{\bar{\alpha}}^{\dagger}(t) d_{\bar{\alpha}}(t)\right.\right.$ $\left.\left.\times d_{\alpha}(t)\right\rangle\right\}(d t / 2 \pi)$. The quantity $i_{\alpha}(\epsilon)$ can be viewed as an intradot "vertical" current density" at energy $\epsilon$ that is contributed by carriers with other energies due to the $e$ - $e$ scattering process. Summing over the index $\alpha$, Eq. (3) reduces to $\Sigma_{n} j_{n}(\epsilon)+\Sigma_{\alpha} i_{\alpha}(\epsilon)=0$, which is exactly the steady-state current conservation equation so that the total current density-including the vertical flow-through the intradot energy level $\epsilon$ is zero. If $G_{\alpha}^{r}(\epsilon)$ and $i_{\alpha}(\epsilon)$ have been solved, from Eq. (3) $G_{\alpha}^{<}(\epsilon)$ can be obtained immediately.

Next, we solve the vertical flow quantity $i_{\alpha}(\epsilon)$. We introduce two-particle contour-ordered Green's functions $B_{\alpha}(t, 0)$ and $\quad B_{c}(t, 0), B_{\alpha}(t, 0) \equiv-\left\langle T_{C}\left[d_{\alpha}(t) d_{\bar{\alpha}}\left(0^{+}\right) d_{\alpha}^{\dagger}(0) d_{\bar{\alpha}}^{\dagger}(0)\right]\right\rangle$ and $\quad B_{c}(t, 0) \equiv-\left\langle T_{C}\left[d_{\alpha}(t) d_{\bar{\alpha}}(t) d_{\alpha}^{\dagger}(0) d_{\bar{\alpha}}^{\dagger}(0)\right]\right\rangle$. Although there are four operators in the definition $B_{\alpha / c}(t, 0)$, only two time indices at $(t, 0)$ appear. We can therefore write the contour- ordered quantities as $B_{\alpha / c}^{++}(t, 0), \quad B_{\alpha / c}^{+-}(t, 0)$, $B_{\alpha / c}^{-+}(t, 0)$, and $B_{\alpha / c}^{--}(t, 0)$. Their Fourier transformations can be defined as $B(\epsilon) \equiv \int e^{i \epsilon t} B(t, 0) d t$. Using the two-particle Green's function, the vertical flow $i_{\alpha}(\epsilon)$ is reduced to $i_{\alpha}(\epsilon)=-(e U / \pi) \operatorname{Im} B_{\alpha}^{+-}(\epsilon)$.

The contour Green's function $B_{\alpha / c}$ is solved by means of a Feynman diagram expansion using the Wick's theorem. We

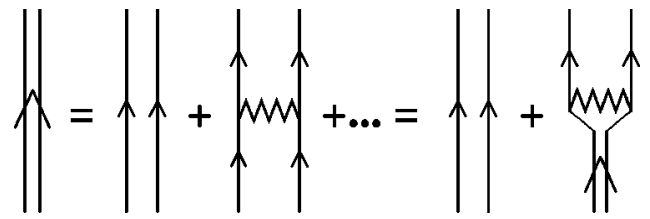

FIG. 1. Relevant Feynman diagrams considered in this work. The single solid lines, double solid lines, and the wave lines stand for the Green's functions $G_{\alpha}, B_{\alpha / c}$, and interaction $U$, respectively.

take the interacting part of the Hamiltonian as $H_{I}$ $=U d_{\uparrow}^{\dagger} d_{\uparrow} d_{\downarrow}^{\dagger} d_{\downarrow}$, and the remaining part $H-H_{I}$ as the noninteracting Hamiltonian $H_{0}$. The first-order irreducible selfenergy we consider is shown in Fig. 1. It is clear that the zeroth order graph describes a two-particle independent propagation process without scattering. The first-order graph describes a two-particle propagation involving the exchange of an interacting energy $U$. Similarly, higher-order graphs (which are reducible) consist of the two-particle propagation process with multiple exchanges of $U$. These graphs add up to the vertex shown in the last diagram of Fig. 1. Two important points should be mentioned here. (i) The vertex we calculate sums up all reducible diagrams constructed by the irreducible self-energy of Fig. 1 [also see Eq. (4)]. This level of approximation is equivalent to that of a typical randomphase approximation, i.e., we compute the irreducible selfenergy up to order $U^{-1}$. It is reasonable to neglect other higher-order irreducible diagrams which are of orders $U^{-2}$ and higher, because the interaction energy $U$ is large. (ii) Single solid lines in Fig. 1 stand for the intradot contourordered Green's functions $G_{\alpha}$ of the Hamiltonian $H$ (not $H_{0}$ ). This means that we have summed over all terms in the Feynman diagram expansion of $G_{\alpha}$.

The corresponding equation for Fig. 1 is

$$
\begin{aligned}
B_{c / \alpha}(t, 0)= & -G_{\alpha}(t, 0) G_{\bar{\alpha}}\left(t / 0^{+}, 0\right) \\
& +i U \int_{C} d t_{1} G_{\alpha}\left(t, t_{1}\right) G_{\bar{\alpha}}\left(t / 0^{+}, t_{1}\right) B_{c}\left(t_{1}, 0\right) .
\end{aligned}
$$

Finally, we get $B_{c}^{--}(t, 0), B_{c}^{+-}(t, 0)$, and $B_{\alpha}^{+-}(t, 0)$ from Eq. (4), and upon taking a Fourier transformation we get

$$
\begin{aligned}
B_{c}^{--}(\epsilon)= & -\int \frac{d \omega}{2 \pi} G_{\alpha}^{--}(\epsilon-\omega) G_{\bar{\alpha}}^{--}(\omega) \\
& +i U\left[B_{c}^{+-}(\epsilon) \int \frac{d \omega}{2 \pi} G_{\alpha}^{-+}(\epsilon-\omega) G_{\bar{\alpha}}^{-+}(\omega)\right. \\
& \left.-B_{c}^{--}(\epsilon) \int \frac{d \omega}{2 \pi} G_{\alpha}^{--}(\epsilon-\omega) G_{\bar{\alpha}}^{--}(\omega)\right], \\
B_{c}^{+-}(\epsilon)= & -\int \frac{d \omega}{2 \pi} G_{\alpha}^{+-}(\epsilon-\omega) G_{\bar{\alpha}}^{+-}(\omega) \\
& +i U\left[B_{c}^{+-}(\epsilon) \int \frac{d \omega}{2 \pi} G_{\alpha}^{++}(\epsilon-\omega) G_{\bar{\alpha}}^{++}(\omega)\right. \\
& \left.-B_{c}^{--}(\epsilon) \int \frac{d \omega}{2 \pi} G_{\alpha}^{+-}(\epsilon-\omega) G_{\bar{\alpha}}^{+-}(\omega)\right]
\end{aligned}
$$




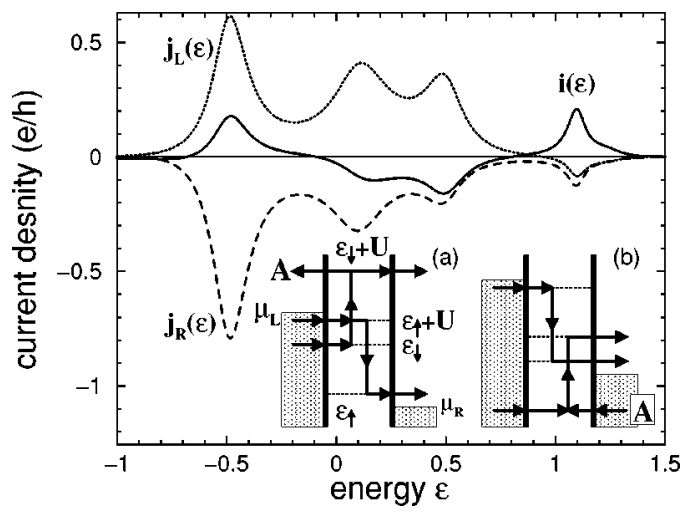

FIG. 2. $j_{L / R}(\epsilon)$ and $i(\epsilon)$ versus energy $\epsilon$ in the wideband limit. The parameters are $\epsilon_{\uparrow}=-0.5, \epsilon_{\downarrow}=0.1, U=1, T=\Gamma=0.1$, and $\mu_{L}$ $=-\mu_{R}=0.7$. Insets (a) and (b) are schematic plots for two kinds of TET processes.

$$
\begin{aligned}
B_{\alpha}^{+-}(\epsilon)= & -i n_{\bar{\alpha}} G_{\alpha}^{+-}(\epsilon) \\
& +i U\left[G_{\alpha}^{++}(\epsilon) \int \frac{d \omega}{2 \pi} G_{\bar{\alpha}}^{-+}(\omega-\epsilon) B_{c}^{+-}(\omega)\right. \\
& \left.-G_{\alpha}^{+-}(\epsilon) \int \frac{d \omega}{2 \pi} G_{\bar{\alpha}}^{--}(\omega-\epsilon) B_{c}^{--}(\omega)\right] .
\end{aligned}
$$

From Eqs. (5)-(7), $B_{\alpha}^{+-}(\epsilon)$ can easily be expressed in terms of $G_{\alpha}^{++}(\epsilon), G_{\alpha}^{+-}(\epsilon), G_{\alpha}^{-+}(\epsilon)$, and $G_{\alpha}^{--}(\epsilon)$-these four Green's functions are directly related to $G_{\alpha}^{r}$ and $G_{\alpha}^{<}$. This completes the analytical derivations.

From Eqs. (2), (3), and (5)-(7), the intradot occupation number $n_{\alpha}$ and the vertical flow $i_{\alpha}(\epsilon)$ are determined selfconsistently. Let's consider a two-probe $(n=L, R)$ device and the wideband approximation in which $\Gamma_{n}(\epsilon)$ is independent of energy $\epsilon$. Figure 2 plots the vertical flow $i(\epsilon)$ $=\Sigma_{\alpha} i_{\alpha}(\epsilon)$ (solid line) as well as the current density $j_{L / R}(\epsilon)$ at a high bias. Clearly, the vertical flow $i(\epsilon)$ is nonzero due to the TET processes, and its value $|i(\epsilon)|$ has four peaks at energies $\epsilon_{\uparrow}, \epsilon_{\downarrow}, \epsilon_{\uparrow}+U$, and $\epsilon_{\downarrow}+U$, respectively. This means that the incident electron indeed can vary its energy in QD by $e-e$ scattering. In contrast, in a typical SET process the electron keeps its energy and does not induce any vertical flow $i(\epsilon)$.

The physics of the TET process that induces the vertical flow is shown in the inset of Fig. 2(a). To start, two incident electrons from the left lead having energies $\epsilon_{\downarrow}$ and $\epsilon_{\uparrow}+U$ tunnel into the QD. They scatter with each other inside the QD and exchange energy to final states $\epsilon_{\uparrow}$ and $\epsilon_{\downarrow}+U$. Afterwards they tunnel out of the QD. In the vertical flow curve of Fig. 2, two peaks at $\epsilon_{\downarrow}$ and $\epsilon_{\uparrow}+U$ are negative (dips), and the other two peaks at $\epsilon_{\uparrow}$ and $\epsilon_{\downarrow}+U$ are positive, precisely indicating the transfer of states from the initial ones at $\epsilon_{\downarrow}$ and $\epsilon_{\uparrow}+U$ to the final ones at $\epsilon_{\uparrow}$ and $\epsilon_{\downarrow}+U$. It is also worth mentioning that besides the new TET process, the usual SET processes also exist in charge transport through the QD in the present case. These SET processes manifest themselves as the three positive (negative) peaks emerging at $\epsilon_{\uparrow}, \epsilon_{\downarrow}$, and $\boldsymbol{\epsilon}_{\uparrow}+U$ in the current-density curves $j_{L}(\boldsymbol{\epsilon})\left[j_{R}(\boldsymbol{\epsilon})\right]$. These processes correspond to single-electron tunneling from the left to the right leads through resonance at $\epsilon_{\uparrow}, \epsilon_{\downarrow}$, and $\epsilon_{\uparrow}$ $+U$, respectively.

So far we have demonstrated that TET processes can exist. In the following we analyze several important questions concerning TET. What is its consequence? (i) TET redistributes the current over energies, and it makes current density a nonconserved quantity, i.e., $\Sigma_{n} j_{n}(\epsilon) \neq 0$. Of course, the total current is still conserved, i.e., $\Sigma_{n} I_{n}=0$. This can be easily proved from the definition of the vertical flow $i(\epsilon)$, namely, $i(\epsilon)$ has the property $\int i(\epsilon) d \epsilon=0$. (ii) TET can involve high-energy empty states, namely, states which are higher, by about $U$, than the highest chemical potential $\max \left(\mu_{L}, \mu_{R}\right)$ [see the inset of Fig. 2(a)]; it may also involve electrons deep inside the Fermi sea, namely, states which are lower, by about $U$, than the lowest chemical potential $\min \left(\mu_{L}, \mu_{R}\right)$ [see the TET process shown in the inset of Fig. 2(b)]. (iii) TET may induce a negative current density at the highvoltage terminal, i.e., the left lead. This means that particles can tunnel from the QD to the high-voltage terminal [indicated by the negative peak at $\epsilon_{\downarrow}+U$ in the $j_{L}(\epsilon)$ curve, and by the arrow "A" in the inset of Fig. 2(a)]. Similarly, TET may also induce a positive current density at the low-voltage terminal, i.e., the right lead [see arrow " $A$ " in the inset of Fig. 2(b)]. These characteristics are rather different from the typical elastic SET processes.

Under what conditions does TET or the vertical flow $i(\epsilon)$ exist? (i) We found that an increase (decrease) of temperature $T$ or linewidth $\Gamma$ will widen (narrow) the peaks of vertical flow, but does not affect peak positions and heights significantly. (ii) If $U=0$, the vertical flow $i(\epsilon)=0$ identically: TET crucially depends on this parameter. (iii) If $U$ $\rightarrow \infty, i(\epsilon)$ tends to zero. This is because at large $U$, the intradot two-electron occupation is prohibited, therefore TET is blocked. In this case only SET processes occur. (iv) When bias potential $e V=\mu_{L}-\mu_{R}$ is less than $U, i(\epsilon)$ decreases drastically. In the limit of $e V=0, i(\epsilon)=j_{n}(\epsilon)=0$.

Are observable quantities of charge transport affected by the TET process? (i) Clearly the current density $j_{L / R}(\epsilon)$ is redistributed significantly, as already discussed above. (ii) In general, the current, conductance, and $n_{\alpha}$ will be affected significantly by TET (see below). However, if one uses the wideband approximation, the TET dependence in charge current will be lost.

In the rest of this paper, we apply the property of TET to design a device so that electrons can be pumped from a lead with lower chemical potential to another lead having a higher chemical potential. Consider a device with three leads ( $n$ $=1,2,3$ ) and consider the nonwideband case. We use a model of quasisquare bands in which the coupling $\Gamma_{n}(\epsilon)$ $=\Gamma /\left\{\exp \left[\left(\left|\epsilon-c_{n}\right|-W\right) / 0.05\right]+1\right\}$; the width of the band is set by $2 W=1$; and its center at $c_{n}$, which is dependent on the terminal voltage $e V_{n}=\mu_{n}$ but $\mu_{n}-c_{n}$, is kept fixed. More specifically, let's assume lead 1 to be a p-type semiconductor with $\mu_{1}-c_{1}=0.4$, lead 2 is an $n$-type semiconductor with $\mu_{2}-c_{2}=-0.4$, and lead 3 is a metal with $\mu_{3}$ $-c_{3}=0$ (see Fig. 4). ${ }^{7}$ The energy diagram of the device is set by external voltages as that shown in Fig. 4 below so that 


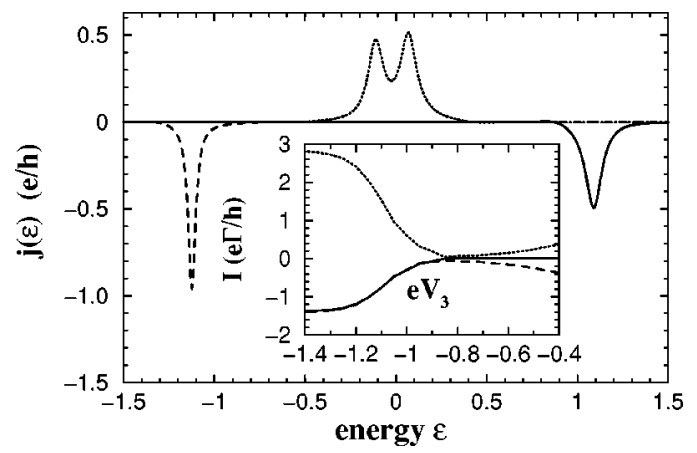

FIG. 3. The main plot shows $j_{n}(\epsilon)$ versus $\epsilon$ with $\mu_{3}=-1.3$ and the inset shows $I_{n}$ versus $e V_{3}=\mu_{3}$ for the nonwideband case. Other parameters $\quad$ are $\quad \epsilon_{\uparrow}=-1.1, \epsilon_{\downarrow}=-0.1, U=1.2, T=\Gamma=0.05, \mu_{1}$ $=0.4$, and $\mu_{2}=0.9$. The dotted, solid, and dashed curves in the main plot correspond to $j_{1}(\epsilon), j_{2}(\epsilon)$, and $j_{3}(\epsilon)$, respectively; they correspond to $I_{1}, I_{2}$, and $I_{3}$ in the inset.

$\mu_{2}>\mu_{1}>\mu_{3}$. The current density $j_{n}(\epsilon)$ in this case is shown in Fig. 3. We note that $j_{1}(\epsilon)$ (dotted line) has two positive peaks at $\epsilon_{\downarrow}$ and $\epsilon_{\uparrow}+U ; j_{2}(\epsilon)$ and $j_{3}(\epsilon)$ each have one negative peak at $\epsilon_{\downarrow}+U$ and $\epsilon_{\uparrow}$, respectively; and $i(\epsilon)$ $=-\Sigma_{n} j_{n}(\epsilon)$ has two negative peaks at $\epsilon_{\downarrow}$ and $\epsilon_{\uparrow}+U$ and two positive peaks at $\epsilon_{\downarrow}+U$ and $\epsilon_{\uparrow}$. The current $I_{n}$ $=\int j_{n}(\epsilon) d \epsilon$ is quite large. We emphasize two points for this pump. (i) In this device the SET process almost does not occur because bands of different leads do not overlap. Then, clearly, the large current $I_{n}$ originates from the TET process: $I_{n} \rightarrow 0$ if the vertical flow $i(\epsilon) \rightarrow 0$. This demonstrates that TET can significantly affect charge current in the general case of nonwideband coupling. (ii) The charge current in the terminal with the highest bias voltage, e.g., lead 2, is negative (solid line in the inset of Fig. 3), which demonstrates the pump effect. The pumps works because when an electron tunnels from lead 1 to 3 , it emits energy $U$ to pump another electron from lead 1 to lead 2, through the TET process.

More clearly, the working principle of the TET pump is summarized in Fig. 4. (i) We start from a situation in which no charge is in the QD so that levels $\epsilon_{\uparrow}$ and $\epsilon_{\downarrow}$ are empty. In this situation, an electron in the Fermi sea of lead 1 having energy $\epsilon_{\downarrow}$ can easily tunnel into the QD [Fig. 4(a)]. (ii) After this electron tunnels into the QD and occupies the QD level of $\epsilon_{\downarrow}$, the other intradot level $\epsilon_{\uparrow}$ is raised to $\epsilon_{\uparrow}+U$, so that another electron with energy $\epsilon_{\uparrow}+U$ in lead 1 tunnels into the QD [Fig. 4(b)]. (iii) When the second electron comes into the $\mathrm{QD}$, due to the $e-e$ Coulomb interaction $U$, the level $\epsilon_{\downarrow}$ with its electron is raised to $\epsilon_{\downarrow}+U$, leading to a negative peak at $\epsilon_{\downarrow}$ and a positive peak at $\epsilon_{\downarrow}+U$ in the vertical flow curve $i(\epsilon)$. Now the intradot two-electron system has total energy $\epsilon_{\uparrow}+\epsilon_{\downarrow}+U$ [Fig. 4(c)]. Afterwards the first electron in state $\epsilon_{\downarrow}+U$ easily tunnels to lead 2 and takes away energy $\epsilon_{\downarrow}$ $+U$. The net effect is that the two electrons exchanged energy $U$, which is the TET process discussed above. When the first electron leaves the QD, the other electron at $\epsilon_{\uparrow}+U$ falls to $\epsilon_{\uparrow}$, leading to a negative peak at $\epsilon_{\uparrow}+U$ and a positive peak at $\epsilon_{\uparrow}$ in the curve of $i(\epsilon)$. (iv) Finally, the second electron tunnels to lead 3 [Fig. 4(d)] and our device returns to its initial conformation of Fig. 4(a). This way an electron is
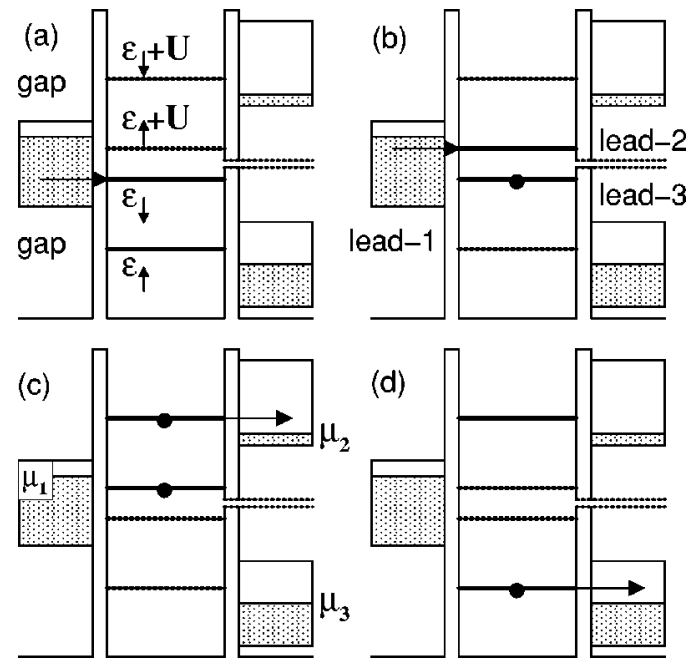

FIG. 4. Schematic plots for the working principle of the TET charge pump.

pumped from lead 1 to lead 2 , where $\mu_{1}<\mu_{2}$, via the TET process. We emphasize that each tunneling event from Fig. 4(a)-4(d) is a first-order normal tunneling event in which tunneling occurs at two aligning states (not like a higherorder virtual cotunneling process). ${ }^{8}$ We therefore conclude that the TET process should have large a probability to occur so that $i(\epsilon)$ can be near the unit value $e / h$ (see Fig. 3). We have also investigated the terminal voltage (e.g., $V_{3}$ ) dependence of current $I_{n}$, shown in the inset of Fig. 3. As $V_{3}$ is increased so that $e V_{3}=\mu_{3}$ passes the lowest resonance state $\epsilon_{\uparrow}$, the tunneling event in Fig. 4(d) cannot occur and the TET process is blocked, leading to a significant reduction of all currents $I_{n}$ (including the pumping current $I_{2}$ ) (see inset of Fig. 3).

In summary, we have investigated the two-electron correlated scattering process in a mesoscopic system. TET induces a vertical flow in the scattering region so that electrons enter and exit the device with different energies. TET is found to redistribute the current over energies, and the process can involve high empty states and/or low filled states of the leads. The properties of TET suggests an interesting working principle of an electron pump which pumps charge carried from a lead with low chemical potential to another lead with a higher chemical potential. Our proposed pump is very different when compared to the electron-photon or parametric pumps. ${ }^{9,10}$ It is also worth mentioning that it is not necessary to use $n$ - and $p$-type semiconductors and a metal in an experimental setup. If the bands of lead 1 are full and bands of leads 2 and 3 are empty, the electron pump will still work. Therefore it should be experimentally feasible to realize the device in a two-dimensional electron gas. In that case, the narrow bands in our theory can be replaced with Landau levels.

We gratefully acknowledge financial support from NSERC of Canada, FCAR of Quebec (Q.-f.S. and H.G), the National Science Foundation of China, the Chinese Academy of Sciences (Q.-f.S.), and a RGC grant from the SAR Government of Hong Kong under Grant No.HKU 7091/01P (J.W.). 
${ }^{1}$ S. Datta, Electronic Transport in Mesoscopic Systems (Cambridge University, Cambridge, England, 1995); H. Haug and A.-P. Jauho, Quantum Kinetics in Transport and Optics of Semiconductors (Springer-Verlag, Berlin, 1998).

${ }^{2}$ Y. Meir and N.S. Wingreen, Phys. Rev. Lett. 68, 2512 (1992).

${ }^{3}$ Q.-f. Sun and H. Guo, Phys. Rev. B 64, 153306 (2001).

${ }^{4}$ Y. Meir, N.S. Wingreen, and P.A. Lee, Phys. Rev. Lett. 66, 3048 (1991); ibid. 70, 2601 (1993).

${ }^{5}$ T.-K. Ng, Phys. Rev. Lett. 76, 487 (1996).

${ }^{6}$ N.S. Wingreen and Y. Meir, Phys. Rev. B 49, 11040 (1994).

${ }^{7}$ Note that it is not necessary to use $n$ - and $p$-type semiconductors and a metal in an experiment. In fact, if the bands of lead 1 are full and bands of leads 2 and 3 are empty, the results are the same.

${ }^{8}$ S. De Franceschi, S. Sasaki, J.M. Elzerman, W.G. van der Wiel, S. Tarucha, and L.P. Kouwenhoven, Phys. Rev. Lett. 86, 878 (2001).

${ }^{9}$ C.A. Stafford and N.S. Wingreen, Phys. Rev. Lett. 76, 1916 (1996); T.H. Oosterkamp, T. Fujisawa, W.G. van der Wiel, K. Ishibashi, R.V. Hijman, S. Tarucha, and L.P. Kouwenhoven, Nature (London) 395, 873 (1998).

${ }^{10}$ Y. Wei, J. Wang, and H. Guo, Phys. Rev. B 62, 9947 (2000); P. Sharma and C. Chamon, Phys. Rev. Lett. 87, 096401 (2001). 